\title{
Reliability and validity of heart rate variability threshold assessment during an incremental shuttle-walk test in middle-aged and older adults
}

\author{
V.Z. Dourado and R.L.F. Guerra \\ Laboratório de Estudos da Motricidade Humana, Departamento de Ciências do Movimento Humano, \\ Universidade Federal de São Paulo, Santos, SP, Brasil
}

\begin{abstract}
Studies on the assessment of heart rate variability threshold (HRVT) during walking are scarce. We determined the reliability and validity of HRVT assessment during the incremental shuttle walk test (ISWT) in healthy subjects. Thirty-one participants aged $57 \pm 9$ years (17 females) performed 3 ISWTs. During the 1 st and 2 nd ISWTs, instantaneous heart rate variability was calculated every $30 \mathrm{~s}$ and HRVT was measured. Walking velocity at HRVT in these tests (WV-HRVT1 and WV-HRVT2) was registered. During the 3rd ISWT, physiological responses were assessed. The ventilatory equivalents were used to determine ventilatory threshold (VT) and the WV at VT (WV-VT) was recorded. The difference between WV-HRVT1 and WV-HRVT2 was not statistically significant (median and interquartile range $=4.8 ; 4.8$ to $5.4 \mathrm{vs} 4.8 ; 4.2$ to $5.4 \mathrm{~km} / \mathrm{h}$ ); the correlation between WV-HRVT1 and WV-HRVT2 was significant $(r=0.84)$; the intraclass correlation coefficient was high $(0.92 ; 0.82$ to 0.96$)$, and the agreement was acceptable $(-0.08 \mathrm{~km} / \mathrm{h} ;-0.92$ to 0.87$)$. The difference between WV-VT and WV-HRVT2 was not statistically significant $(4.8 ; 4.8$ to $5.4 \mathrm{vs} 4.8 ; 4.2$ to $5.4 \mathrm{~km} / \mathrm{h})$ and the agreement was acceptable $(0.04 \mathrm{~km} / \mathrm{h} ;-1.28$ to 1.36$)$. HRVT assessment during walking is a reliable measure and permits the estimation of VT in adults. We suggest the use of the ISWT for the assessment of exercise capacity in middle-aged and older adults.
\end{abstract}

Key words: Heart rate variability; Exercise; Walking; Adults

\section{Introduction}

The determination of heart rate variability (HRV) consists of measuring the RR intervals of heart beats and calculating their variation (1). During exercise, the increase in intensity results in an increase in heart rate (HR) and reduction in HRV (2). Vagal modulation of HR disappears almost completely at approximately 50 to $60 \%$ oxygen uptake at the end of incremental exercise (peak $\mathrm{VO}_{2}$ ); thereafter, the increase in HR is mainly mediated by sympathetic activation (2). The HRV threshold (HRVT) occurs at this intensity and is characterized as the instant at which the HRV no longer undergoes excessive variation due to the increase in exercise intensity (2). Currently, HRVT can be easily obtained using a commercial heart monitor. Accordingly, HRVT may be useful as a simple, inexpensive tool for prescribing exercise at an appropriate intensity.

The validity of HRVT in estimating the ventilatory threshold (VT) has been recently confirmed both in field running and walking tests, with HRVT and VT identified at the same exercise intensity $(3,4)$. Specifically during walking, Dourado et al. (3) demonstrated the usefulness of the incremental shuttle walk test (ISWT) for such purpose in healthy adults. The ISWT was developed to assess the functional exercise capacity of patients with respiratory diseases (5). The test consists of walking in a $10-\mathrm{m}$ flat hallway at progressive velocities imposed by audio signals until exhaustion. The incremental character of the ISWT yields physiological responses closer to those observed in cardiopulmonary exercise testing performed in the laboratory (6).

The ISWT was related to a modest learning effect in previous studies involving patients and healthy subjects $(6,7)$. Jurgensen et al. (7) found a statistically significant mean difference of about $40 \mathrm{~m}$ between the distance achieved in the first ISWT and that achieved in the second $(P=0.043)$. This finding underscores the need for 
familiarization with the ISWT in order to avoid the misinterpretation of functional exercise capacity (8).

Walking is a popular form of physical exercise for the conditioning of middle-aged and elderly individuals (9). In some clinical situations, exercise performed at a greater intensity and/or volume is recommended $(10,11)$. Thus, simple strategies for the determination of ideal walking intensity are welcome. In healthy subjects, the VT has been properly estimated using HRVT during the ISWT (3). HRVT assessed during walking constitutes a promising strategy for prescribing exercise in health promotion programs and clinical settings. However, since the ISWT is related to a significant learning effect, the reliability of assessment of HRVT obtained during two subsequent ISWTs should be investigated.

The aim of the present study was to evaluate the reliability of HRVT measurement in middle-aged and older adults and to determine the validity of HRVT for estimating VT.

\section{Material and Methods}

\section{Participants}

Forty participants older than 40 years were invited to participate in the present study. All subjects were free of cardiac and pulmonary disease. Current and former smokers and those with a body mass index (BMI) $>35 \mathrm{~kg} / \mathrm{m}^{2}$ were excluded from the study. The participants were asked to avoid the use of any substance capable of altering the autonomic modulation of HR (e.g., alcohol). Following enrollment, the participants were informed about the procedures, potential discomfort and risks associated with the study and all gave written informed consent. The present study was approved by the Ethics Committee of Universidade Federal de São Paulo (CEP 1525/08). The participants underwent evaluations related to the protocol in the morning of 2 consecutive days in the same week. Screening and anthropometric evaluations were performed on the first day and cardiopulmonary fitness was evaluated through three ISWTs on the second day, with at least 20-min intervals between tests. After the first walk, the subsequent tests were applied only after cardiopulmonary recovery to basal values so that the exercise-induced sympathetic activity could be minimized.

\section{Incremental shuttle walk test}

The ISWT was conducted on the basis of the method described by Singh et al. (5) in a 10-m hallway delimitated by 2 traffic cones at progressive speeds (increase of 0.17 $\mathrm{m} / \mathrm{s}$ every minute) that was controlled by a series of sounds. The start of the test was indicated by a 3-s sound. Thereafter, short regular beeps indicated the moment that the participants should be turning around the cone. Every minute, the walking velocity increased according to a different sound (more prolonged). When the subject was unable to reach the closest cone (i.e., was $>0.5 \mathrm{~m}$ from the cone) by the time the signal sounded, the rater ended the test, which also could have been terminated by the subject for any reason. Three tests were performed 20 min apart. The original protocol (12 levels) was slightly modified by extending the test up to 15 levels, since the present study involved only healthy subjects. Distance in meters and walking velocity (WV) in $\mathrm{km} / \mathrm{h}$ were selected for further analysis. HRV was assessed during the first and second ISWTs and gas exchange analysis was performed in the third test.

\section{Physiologic responses}

During the third ISWT, expired gases were collected through a mask and measured using a portable telemetric gas analyzer $\left(\mathrm{K} 4 \mathrm{~b}^{2}\right.$, Cosmed, Italy). The participants breathed through a face mask with minimal dead space $(0.30 \mathrm{~mL})$. The gas analyzer (total weight: $<1 \mathrm{~kg}$ ) was attached to the trunk of the participant with straps over his shoulders, allowing free movement. The reference gas, 3-L syringe and delay calibrations were used following manufacturer recommendations. Among other variables, oxygen uptake $\left(\mathrm{VO}_{2}\right)$, carbon dioxide production $\left(\mathrm{VCO}_{2}\right)$ and ventilation (VE) were monitored breath-by-breath throughout the test. The data were filtered every $30 \mathrm{~s}$ and the arithmetic mean of the last $30 \mathrm{~s}$ of the ISWT was used as representative of peak values.

\section{Threshold assessment}

To determine VT, the ventilatory equivalents of $\mathrm{O}_{2}$ $\left(\mathrm{VE} / \mathrm{VO}_{2}\right)$ and $\mathrm{CO}_{2}\left(\mathrm{VE} / \mathrm{VCO}_{2}\right)$ were plotted in relation to exercise intensity (i.e., WV) based on the methods described by Wasserman et al. (12). VT was defined as the first non-linear increment in the $\mathrm{VE} / \mathrm{VO}_{2}$ curve, while the $\mathrm{VE} / \mathrm{VCO}_{2}$ values remained constant. $\mathrm{VO}_{2}$ obtained at $\mathrm{VT}\left(\mathrm{VO}_{2} \mathrm{VT}\right)$ was quantified. $\mathrm{WV}$ at $\mathrm{VO}_{2} \mathrm{VT}(\mathrm{WV}-\mathrm{VT})$ was also recorded for further analysis.

The RR intervals at rest and each $60 \mathrm{~s}$ of the ISWT were used to assess HRV. The data were automatically filtered using the Polar ProTrainer 5 software (Polar Electro $^{\mathrm{TM}}$, Finland) and then visually inspected. Inadequate heart beats were corrected by interpolating data derived from adjacent normal RR intervals. Any RR interval with a difference greater than $20 \%$ of the previous interval was automatically and visually filtered. For the determination of HRVT, the values of the instantaneous variability in the RR intervals (SD1) from the Poincaré plot were plotted against WV. HRVT was assessed in the first stage of exercise in which the difference between the instantaneous variability in the RR intervals (SD1) of two consecutive stages was less than $1 \mathrm{~ms}$ and no longer changed significantly. WV at HRVT in the first (WVHRVT1) and second ISWT (WV-HRVT2) was obtained for further analysis.

\section{Statistical analysis}

The individuals were characterized by descriptive 
analysis of the data. The Student t-test, Pearson's correlation, intraclass correlation coefficient (ICC), and Bland and Altman plot analysis for comparing values obtained in the first and second ISWTs were used to determine the reliability of HRVT. Except for the ICC, the same statistical methods were used for assessing the validity of HRVT in estimating VT regarding the values obtained in the second and third ISWTs. For all statistical tests, $P$ values less than 0.05 were considered to be significant. Statistical analysis was performed using SigmaStat 3.1 (Systat Software Inc. ${ }^{\mathrm{TM}}$, USA) and MedCalc version 11.1.1 (MedCalc Software ${ }^{\mathrm{TM}}$, Belgium) statistical packages.

\section{Results}

It was not possible to measure HRVT in 9 of the 31 participants at the beginning of the study due to mechanical artifacts in the heart monitor $(n=2)$ and a lack of satisfactory exercise intensity $(n=7)$, with the peak HR below $80 \%$ of the maximum estimated (220 minus age). The general characteristics of these participants ( 5 females; $58 \pm 10$ years; $27 \pm 4 \mathrm{~kg} / \mathrm{m}^{2}$ ) were not statistically different from those of the remaining sample. Thus, 31 participants composed the final sample of the present study (Table 1), 9 of whom had a BMI between 30 and $35 \mathrm{~kg} / \mathrm{m}^{2}$.

The ISWT represented high-intensity exercise, as the $\mathrm{HR}$ at the end of the test corresponded to $88 \pm 10 \%$ of the maximum. Mean peak $\mathrm{VO}_{2}, \mathrm{VCO}_{2}$ and $\mathrm{VE}$ reached $1845 \pm 508,1843 \pm 507$, and $55 \pm 19 \mathrm{~mL} / \mathrm{min}$, respectively.

HRVT assessment exhibited excellent reliability based on the following results: the difference between WVHRVT1 and WV-HRVT2 was not significant (median and interquartile range $=4.8 ; 4.8$ to 5.4 vs $4.8 ; 4.2$ to $5.4 \mathrm{~km} /$ h); WV-HRVT1 and WV-HRVT2 were strongly correlated $(r=0.84 ; \mathrm{P}<0.0001)$; the agreement between WVHRVT1 and WV-HRVT2 was acceptable (Figure 1); the

Table 1. General characteristics of the individuals studied.

\begin{tabular}{lc}
\hline General characteristics & \\
\hline Gender (male/female) & $14 / 17$ \\
Age (years) & $57 \pm 9$ \\
Height $(\mathrm{m})$ & $1.62 \pm 0.08$ \\
Body mass $(\mathrm{kg})$ & $76 \pm 14$ \\
BMI $\left(\mathrm{kg} / \mathrm{m}^{2}\right)$ & $28 \pm 3$ \\
ISWD $(\mathrm{m})$ & $557 \pm 128$ \\
ISWD $(\%$ predicted $)$ & $114 \pm 18$ \\
Peak WV $(\mathrm{km} / \mathrm{h})$ & $6.55 \pm 0.68$ \\
Peak $\mathrm{VO}_{2}(\mathrm{~mL} / \mathrm{min})$ & $1845 \pm 508$ \\
\hline
\end{tabular}

Data are reported as means $\pm \mathrm{SD}$. BMI = body mass index; ISWD = incremental shuttle walking distance; $\mathrm{WV}=$ walking velocity; $\mathrm{VO}_{2}=$ oxygen uptake.
ICC was high $($ ICC $=0.92 ; 95 \%$ confidence interval $=$ 0.82-0.96), WV-VT did not differ significantly from WVHRVT2, and the agreement between WV-VT and WVHRVT2 was also acceptable (Figure 2).

\section{Discussion}

The assessment of HRV in response to exercise is a promising area in sports and exercise science. Accordingly, for its use in assessing functional exercise capacity before and after an intervention, its reliability should be acceptable. The present study describes a simple, inexpensive method for assessment of HRVT during a field-walking test and demonstrates that an important index of HRV (SD1) is reliable in middle-aged and older adults.

Despite the learning effect, especially between the first and second ISWT, HRVT remained unchanged and demonstrated excellent reliability in the present study. Although the validity of HRVT against VT has been demonstrated, there are few or no studies in the literature

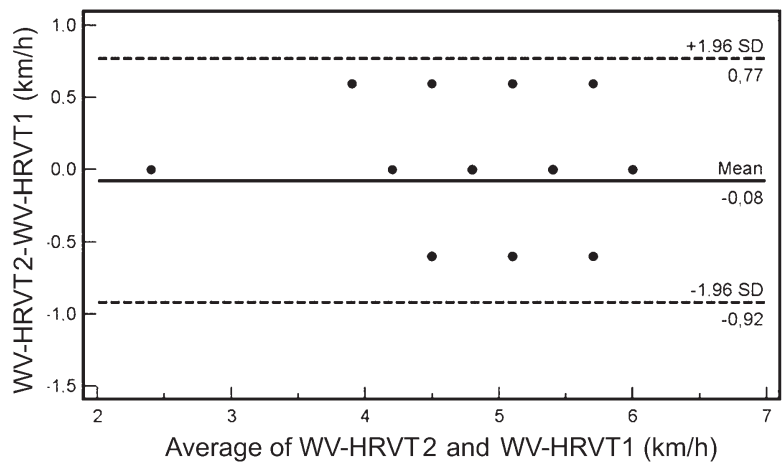

Figure 1. Agreement between walking velocity (WV) at heart rate variability threshold (HRVT) obtained in the 1st (WV-HRVT1) and 2nd (WV-HRVT2) incremental shuttle walk tests.

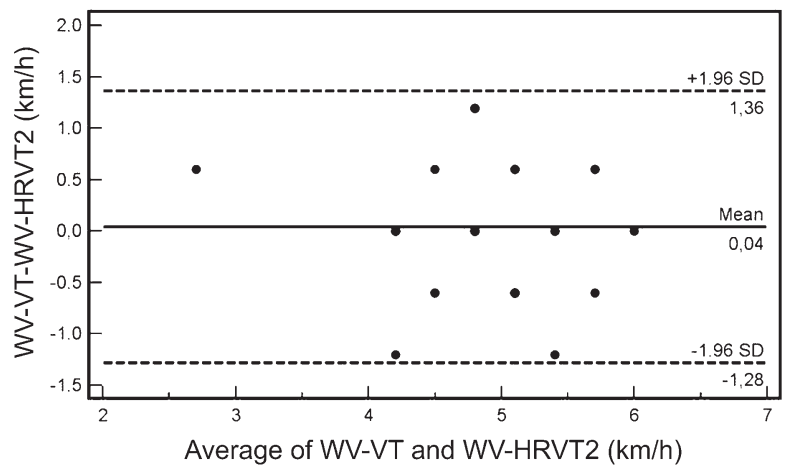

Figure 2. Agreement between walking velocity (WV) at heart rate variability threshold (HRVT) and ventilator threshold (VT) obtained in the 2nd (WV-HRVT2) and 3rd (WV-VT) incremental shuttle walk tests. 
evaluating its reliability. Nevertheless, the present results are in agreement with those of a previously described submaximal exercise. Guijt et al. (13) carried out two measurements of HRV in 26 healthy adults during a cycling test, with a 7-day interval between tests. Reliability was found to be good to excellent for the square root of the mean squared successive difference in RR intervals (RMSSD), with a mean ICC of 0.84 . In a study by Tulppo et al. (2), the confidence interval (95\%) of the difference for SD1 normalized by RR intervals was \pm 5.3 at rest and decreased to \pm 3.3 and \pm 2.2 during 50 and $75 \mathrm{~W}$ of constant exercise, respectively. In fact, some investigators consider reproducibility at rest in both healthy subjects and patients with myocardial infarction to be worse than reproducibility during exercise, as the assessment at rest is potentially influenced by several factors (e.g., time of day, food intake, mental state) $(14,15)$. Nunan et al. (16) used a portable heart monitor to evaluate HRV at rest $(10 \mathrm{~min})$ in healthy subjects and found an ICC of 0.86 for RMSSD. Unfortunately, studies with this objective are quite heterogeneous. A review of the literature reveals that the reliability of the short-term assessment of HRV is controversial, with coefficients of variation ranging from 1 to $100 \%$. Similar results have been reported for the ICC and limits of agreement. Thus, it is premature to affirm that the assessment of HRV at rest is a reliable measure and well-controlled studies are necessary to clarify this topic (17).

Exercise causes a marked alteration in autonomic function, with a gradual vagal withdrawal followed by sympathetic activation (3). The repeated tests performed in the present study demonstrate that the measurement of the vagal modulation of $\mathrm{HR}$ is reliable during an incremental exercise, possibly because the profound effects of the exercise itself on autonomic function minimize the influence of other confounding factors.

The results of the present study confirm the validity of HRVT assessment in estimating VT. These findings are consistent with the literature. Karapetian et al. (18) evaluated 24 healthy adults using a cardiopulmonary exercise test (CPET) performed on a stationary cycle and the results showed agreement and a strong correlation ( $r$ $=0.89$ ) between $\mathrm{VO}_{2}$ at $\mathrm{VT}$ and $\mathrm{VO}_{2}$ at HRVT. Cottin et al. (19) evaluated the correlation between VT and HRVT using the frequency domain of HRV in 11 healthy adults during a CPET on a cycle ergometer and found no significant difference in workload at HRVT and at VT. The correlation between thresholds was intense both in absolute and relative values $(r=0.97$ and 0.92 , respectively).

Few studies have evaluated the usefulness of HRVT to determine VT in field tests. Using the frequency domain of HRV, Cottin et al. (4) evaluated the agreement between HRVT and VT in 12 soccer players during an incremental field running test. VT was evaluated with a telemetric gas analyzer and HRV was obtained using a commercial heart monitor, similar to the methods employed in the present study. There was agreement and strong correlation $\left(\mathrm{R}^{2}=\right.$ 0.94) between running velocities at HRVT and VT. Dourado et al. (3) found similar results during walking (ISWT), reporting statistically similar walking velocities at HRVT and VT. Comparable results have been recently reported for 14 competitive swimmers (20) during five sets of the 200-m front crawl. The trends of the high-frequency HRV spectral power and its fraction relative to the respiratory sinus arrhythmia were analyzed. In all subjects, an abrupt increase in both indices was observed and associated with the lactate threshold. Lactate thresholds respectively determined from lactate concentrations and HRV indices were similar and strongly correlated. HRVT occurs at vagal withdrawal and, in many cases, coincides with the sustained increase in lactate concentration, which is characteristic of the transition between moderate- and high-intensity exercise $(2,12,21)$. In fact, the lactate threshold is mediated by muscle glycogenolysis and the increase in sympathetic activity during intense exercise causes higher concentrations of catecholamines, which may explain the simultaneous increase in ventilation (22).

Considering the peak HR values, the ISWT proved to be a high-intensity exercise. These results reveal the usefulness of the ISWT as a simple way of measuring cardiopulmonary fitness in healthy middle-aged and older adults. Similar results have been obtained with patients with cardiopulmonary diseases $(5,6,23,24)$. Unfortunately, data on the validity of the ISWT for assessing $\mathrm{VO}_{2 \text { peak }}$ in healthy individuals are scarce in the literature. Walking is an important physical activity for health promotion, as it offers fewer physical, social and psychological barriers than other traditional forms of exercise (25). It is socially acceptable and affordable for most people, considering its low cost, relatively low risk and little need for equipment. For sedentary adults, walking represents a moderate intensity physical activity (26). Accordingly, simple, cheap and reliable strategies for prescribing the appropriate amount and intensity of walking, such as that presented here, are useful for increasing the proportion of the population that follows guidelines for physical activity and associated health benefits (9).

The main limitation of the present study was the impossibility of assessing HRVT in about $22 \%$ of the participants. However, as can be seen in the Results section, the demographic and anthropometric characteristics of these participants were similar to those of the remaining sample. Thus, it is reasonable to affirm that the low intensity of walking during the ISWT was the reason for this situation. In fact, a previous study involving 131 healthy individuals who performed two ISWTs showed a substantial variability in distance and HRmax for males and females (7). Motivation before starting the test plays an important role in the total distance traveled in field walking tests (27) and may explain the impossibility of 
assessing HRVT in all participants in the present study. In our sample, there were obese participants, with nine participants having a BMI between 30 and $35 \mathrm{~kg} / \mathrm{m}^{2}$. This feature does not allow us to say that the sample consisted of only healthy individuals. Furthermore, the sample consisted of middle-aged adults and elderly subjects. The increase in body fat and age can affect the cardiovascular autonomic control. Our results should be used with caution in individuals with different demographic and anthropometric characteristics.

The reliability of the assessment of HRVT during an incremental walking test proved to be excellent using

\section{References}

1. Task Force of the European Society of Cardiology and the North American Society of Pacing and Electrophysiology. Heart rate variability: standards of measurement, physiological interpretation and clinical use. Circulation 1996; 93: 1043-1065, doi: 10.1161/01.CIR.93.5.1043.

2. Tulppo MP, Makikallio TH, Seppanen T, Laukkanen RT, Huikuri HV. Vagal modulation of heart rate during exercise: effects of age and physical fitness. Am J Physiol 1998; 274: $\mathrm{H} 424-\mathrm{H} 429$.

3. Dourado VZ, Banov MC, Marino MC, de Souza VL, Antunes LC, McBurnie MA. A simple approach to assess VT during a field walk test. Int J Sports Med 2010; 31: 698-703, doi: 10.1055/s-0030-1255110.

4. Cottin F, Medigue C, Lopes P, Lepretre PM, Heubert R, Billat $V$. Ventilatory thresholds assessment from heart rate variability during an incremental exhaustive running test. Int J Sports Med 2007; 28: 287-294, doi: 10.1055/s-2006924355.

5. Singh SJ, Morgan MD, Scott S, Walters D, Hardman AE. Development of a shuttle walking test of disability in patients with chronic airways obstruction. Thorax 1992; 47: 10191024, doi: 10.1136/thx.47.12.1019.

6. Singh SJ, Morgan MD, Hardman AE, Rowe C, Bardsley PA. Comparison of oxygen uptake during a conventional treadmill test and the shuttle walking test in chronic airflow limitation. Eur Respir J 1994; 7: 2016-2020.

7. Jurgensen SP, Antunes LC, Tanni SE, Banov MC, Lucheta $\mathrm{PA}$, Bucceroni AF, et al. The incremental shuttle walk test in older Brazilian adults. Respiration 2011; 81: 223-228, doi: 10.1159/000319037.

8. Dyer F, Marriner P, Cheema K, Bott J. Is a practice incremental shuttle walk test really necessary? Chron Respir Dis 2011; 8: 201-205, doi: 10.1177/1479972311415128.

9. Murphy MH, Donnelly P, Shibli S, Foster C, Nevill AM. Physical activity, walking and leanness: an analysis of the Northern Ireland Sport and Physical Activity Survey (SAPAS). Prev Med 2012; 54: 140-144, doi: 10.1016/ j.ypmed.2011.12.008.

10. American College of Sports Medicine Position Stand. The recommended quantity and quality of exercise for developing and maintaining cardiorespiratory and muscular fitness, and flexibility in healthy adults. Med Sci Sports Exerc 1998; 30: 975-991, doi: 10.1097/00005768-199806000-00032.

11. Zainuldin R, Mackey MG, Alison JA. Prescription of walking instantaneous HRV. Moreover, the ability of the HRVT to estimate VT during walking was demonstrated. Based on these results, the use of the ISWT is suggested for prescribing the appropriate amount and intensity of exercise in walking programs aimed at promoting health. The HRVT also may be useful for quantifying the effects of different interventions on the autonomic modulation of heart rate.

\section{Acknowledgments}

Research supported by FAPESP (\#2007/08673-3). exercise intensity from the incremental shuttle walk test in people with chronic obstructive pulmonary disease. Am J Phys Med Rehabil 2012; 91: 592-600, doi: 10.1097/ PHM.0b013e31824660bd.

12. Wasserman K, Hansen J, Sue DY, Whipp BJ, Casaburi R. Principles of exercise testing and interpretation. 4th edn. Philadelphia: Lippincott Wiliams \& Wilkins; 2005.

13. Guijt AM, Sluiter JK, Frings-Dresen MH. Test-retest reliability of heart rate variability and respiration rate at rest and during light physical activity in normal subjects. Arch Med Res 2007; 38: 113-120, doi: 10.1016/j.arcmed. 2006.07.009.

14. Breuer HW, Skyschally A, Wehr M, Schulz R, Heusch G. [Poor reproducibility of parameters of heart rate variations] Z Kardiol 1992; 81: 475-481.

15. Maestri R, Raczak G, Szymanowicz L, Torunski A, Sukiennik A, Kubica $J$, et al. Reliability of heart rate variability measurements in patients with a history of myocardial infarction. Clin Sci 2010; 118: 195-201, doi: 10.1042/CS20090183.

16. Nunan D, Donovan G, Jakovljevic DG, Hodges LD, Sandercock GR, Brodie DA. Validity and reliability of short-term heart-rate variability from the Polar S810. Med Sci Sports Exerc 2009; 41: 243-250.

17. Sandercock GR, Bromley PD, Brodie DA. The reliability of short-term measurements of heart rate variability. Int $\mathrm{J}$ Cardiol 2005; 103: 238-247, doi: 10.1016/j.ijcard.2004.09. 013.

18. Karapetian GK, Engels HJ, Gretebeck RJ. Use of heart rate variability to estimate LT and VT. Int J Sports Med 2008; 29: 652-657, doi: 10.1055/s-2007-989423.

19. Cottin F, Lepretre PM, Lopes P, Papelier Y, Medigue C, Billat V. Assessment of ventilatory thresholds from heart rate variability in well-trained subjects during cycling. Int $J$ Sports Med 2006; 27: 959-967, doi: 10.1055/s-2006923849.

20. Michele RD, Gatta G, Leo AD, Cortesi M, Andina F, Tam E, et al. Estimation of the anaerobic threshold from heart rate variability in an incremental swimming test. J Strength Cond Res 2012; 26: 3059-3066, doi: 10.1519/JSC.0b013e318245bde1.

21. Tulppo MP, Makikallio TH, Takala TE, Seppanen T, Huikuri HV. Quantitative beat-to-beat analysis of heart rate dynamics during exercise. Am J Physiol 1996; 271: H244$\mathrm{H} 252$. 
22. Chwalbinska-Moneta J, Robergs RA, Costill DL, Fink WJ. Threshold for muscle lactate accumulation during progressive exercise. J Appl Physiol 1989; 66: 2710-2716.

23. Onorati $P$, Antonucci $R$, Valli G, Berton E, De Marco F, Serra $P$, et al. Non-invasive evaluation of gas exchange during a shuttle walking test vs. a 6-min walking test to assess exercise tolerance in COPD patients. Eur J Appl Physiol 2003; 89: 331-336, doi: 10.1007/s00421-003-08039.

24. Palange $\mathrm{P}$, Forte $\mathrm{S}$, Onorati $\mathrm{P}$, Manfredi F, Serra $\mathrm{P}$, Carlone $S$. Ventilatory and metabolic adaptations to walking and cycling in patients with COPD. J Appl Physiol 2000; 88:
1715-1720.

25. Allender S, Cowburn G, Foster C. Understanding participation in sport and physical activity among children and adults: a review of qualitative studies. Health Educ Res 2006; 21: 826-835, doi: 10.1093/her/cyl063.

26. Murtagh EM, Boreham CA, Murphy MH. Speed and exercise intensity of recreational walkers. Prev Med 2002; 35: 397-400, doi: 10.1006/pmed.2002.1090.

27. Guyatt GH, Pugsley SO, Sullivan MJ, Thompson PJ, Berman L, Jones NL, et al. Effect of encouragement on walking test performance. Thorax 1984; 39: 818-822, doi: 10.1136/thx.39.11.818. 